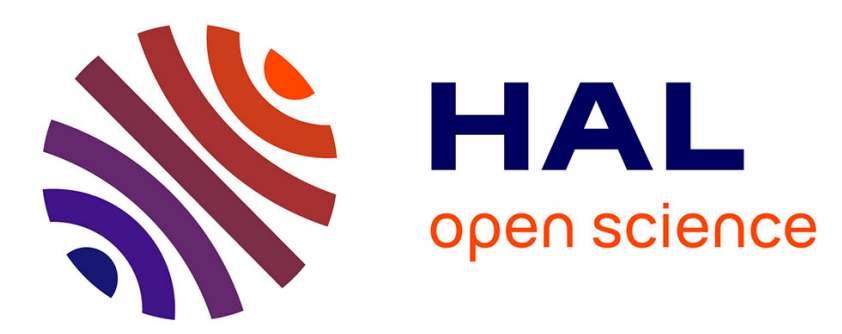

\title{
Patterning of spontaneous rolling thin polymer films for versatile microcapillaries
}

Rémy Brossard, Valeriy Luchnikov, Patrick' Guenoun, Florent' Malloggi

\section{To cite this version:}

Rémy Brossard, Valeriy Luchnikov, Patrick ' Guenoun, Florent' Malloggi. Patterning of spontaneous rolling thin polymer films for versatile microcapillaries. Journal of Polymer Science Part B: Polymer Physics, 2017, 55, pp.721-728. 10.1002/polb.24322 . cea-01483491

\section{HAL Id: cea-01483491 https://hal-cea.archives-ouvertes.fr/cea-01483491}

Submitted on 6 Mar 2017

HAL is a multi-disciplinary open access archive for the deposit and dissemination of scientific research documents, whether they are published or not. The documents may come from teaching and research institutions in France or abroad, or from public or private research centers.
L'archive ouverte pluridisciplinaire HAL, est destinée au dépôt et à la diffusion de documents scientifiques de niveau recherche, publiés ou non, émanant des établissements d'enseignement et de recherche français ou étrangers, des laboratoires publics ou privés. 


\title{
Patterning of spontaneous rolling thin polymer films for versatile microcapillaries
}

\author{
Rémy Brossard ${ }^{1}$, Valériy Luchnikov ${ }^{2}$, Patrick Guenoun ${ }^{1}$, Florent Malloggi ${ }^{1}$
}

\begin{abstract}
We investigate the spontaneous rolling of polydimethylsiloxane(PDMS) thin films and demonstrate the fabrication of capillaries with topographical and chemical patterns on the inner wall. Thin films of polydimethylsiloxane(PDMS) are either coated by a layer of hard material or have their surface hardened by plasma oxidation. They are then driven out of equilibrium by selective solvent swelling in vapor phase resulting in a tubular rolled-up system. The inner diameter of those is measured as a function of layer thickness for different solvents and capping types. Those results are shown to be in good agreement with Timoshenko theory. Before rolling, the future inner surface can be characterized and functionnalized. We demonstrate topographical and chemical patterning, respectively by embossing and microcontact printing. These methods are very simple and can easily produce cylindrical capillaries with inner diameter between 20 and some hundreds of microns with fully functionnalized inner surface, overcoming many difficulties encountered in conventional soft lithography techniques.

Keywords

Patterning, Microfluidics, Microcapillary, Self-rolling, Microfabrication, Polydimethylsiloxane

${ }^{1}$ LIONS, NIMBE, CEA, CNRS, Université Paris-Saclay, CEA Saclay 91191 Gif sur Yvette Cedex, France.

${ }^{2}$ Institut de Science des Matériaux de Mulhouse, UMR 7361 CNRS-UHA, 15 rue Jean Starcky, 68057 Mulhouse
\end{abstract}

\section{Introduction}

Over the recent years, folding has been extensively used as a remarkable microfabrication method for the design of probes and microfluidic devices, leading to the emerging concept of lab-in-a-tube ${ }^{1}$. Advantage was taken from reversible folding in actuator applications such as cell capture, soft robots or cantilever based biosensors $2 ; 3 ; 4$. Initially, metallic and semi-conductor compounds were used. Those systems found applications in many fields such as of photonics ${ }^{5}$ for the design of optical and magnetic resonators $6 ; 7$, microfluidics ${ }^{8 ; 9}$, synthetic neural circuits ${ }^{10 ; 11}$ and energy ${ }^{12}$. However a recent trend and challenge emerged with the fabrication of such system with polymers ${ }^{13 ; 14 ; 15}$ in order to combine the numerous possibilities of chemical functionnalisation and wide range of available mechanical properties of polymeric materials with the easiness of the fabrication process ${ }^{16}$. This approach quickly lead to a fair number of advanced applications in various fields such as tissue engineering ${ }^{17}$, biomimetics ${ }^{18}$ and microtechnology ${ }^{19}$. Spontaneous deformations of thin elastic films triggered by in-plane stress can result processes such as wrinkling ${ }^{20 ; 21}$, creasing ${ }^{22 ; 23}$ but also in self-rolling structures $24 ; 25 ; 13$. The latter are formed in substrate-free films because of a stress gradient along the film thickness. Here we focus on the formation of self-rolled polydimethylsiloxane (PDMS) tubes, as a non-lithographic method to design functionalized microcapillaries. Self-rolled tubes are obtained with diameters between $20 \mu \mathrm{m}$ to $200 \mu \mathrm{m}$, a relevant scale for biology and microfluidics applications. Remarkably, while wrinkling and creasing lead to surface modification of the film ${ }^{26}$, self-rolling is a transformation of a flat surface towards a three dimensional object whose surface is usually unaltered by the process (this aspect is discussed in section 3.2.1). This feature is of primary importance since the surface can be functionalized and fully characterized prior to rolling. So far, most studies reported in the literature focused on electrodes integration ${ }^{13 ; 27 ; 28 ; 29}$ but studies dealing with other kinds of patterning are scarce.

In this paper, we demonstrate the fabrication of tubes with two kinds of inner patterns. Topographic patterning which can be used for example to alter fluid flow - as in micro-mixing applications ${ }^{30}$ - or for its influence on cell growth ${ }^{31 ; 32}$. In this work, this topographical patterning is made by an embossinglike method. Chemical patterning is also an important feature since it can be used to locally alter the wetting behavior, localize cell adhesion or prevent biofouling ${ }^{33}$. As a proof of principle of the latter patterning a fluorescent pattern is fabricated by stamping. In this paper, we also clarify the mechanisms that select the tube size by examining two different bilayer systems. Both are based on a PDMS film of few microns but with different capping layers. In the first case, the surface is hardened by exposure to a low energy oxygen plasma ${ }^{34 ; 29}$ which is a common technique in soft lithography. In the second case, we take benefit of the versatility of using polymers by coating a thin film of a biocompatible polymer: chitosan ${ }^{35}$ is spin coated on PDMS resulting in a film of a few hundreds of nanometers. Rolling occurs when the system is exposed to chloroform ${ }^{29}$ or pentane in gas form. The variation of the ra- 
dius of curvature as a function of the PDMS layer thickness is measured and the predictive ability of standard rolling model is demonstrated for each of our different methods.

\section{Self-rolling instability in a polymer bilayer system}

\subsection{Mechanism of self-rolling}

Mechanical stress in a material can be generated by many stimuli such as thermal expansion, or solvent exposure. In an homogeneous material, this stress is relaxed by shrinking or swelling. However, in an non-homogeneous material this stress leads to fascinating non flat rest positions ${ }^{36 ; 18}$.

The case of bilayer thin films was originally considered by Stoney ${ }^{37}$ and Timoshenko ${ }^{38}$ at the beginning of the twentieth century. The system of interest is a stacking of two perfectly elastic layers. In an initially flat configuration, the top layer is at rest while the bottom layer swells i.e. it has an embedded compressive strain. Such a system is obviously not at equilibrium. Given $L$ the main thickness of the plate, the bending modulus scales as $L^{3}$ while the stiffness scales only as $L$. Hence at small scale, the stress is mainly relaxed by bending. Under small strain, the system will exhibit an isotropic curvature i.e. a sphere portion shape ${ }^{39}$. However due to geometrical constraints, at larger strain, the symmetry is broken and a preferential bending direction is chosen resulting in a cylindrical shaped object.

Complex analytical and numerical models ${ }^{40 ; 41}$ have been developed to understand the interplay of the two dimensions of the system. However, as long as a rolling direction is chosen by the system, we show here that the original simple result of Timoshenko captures well the features of our system as a first approximation. In the regime of small deformation and considering the two directions of curvature as independent, it can be shown that the relation between the diameter $d$, curvature $\kappa=2 / d$ and total thickness of the film $h$, the strain mismatch $\delta$, the ratio of the layers thicknesses $m$ and elastic moduli $n$ is as follows ${ }^{38}$ :

$$
\begin{aligned}
& \kappa=\frac{6 \delta}{h} \frac{(1+m)^{2}}{n m^{3}+n^{-1} m^{-1}+4 m^{2}+6 m+4} \\
& \text { with } m=\frac{h_{f}}{h_{s}} \text { and } n=\frac{E_{f}\left(1-v_{s}\right)}{E_{s}\left(1-v_{f}\right)}
\end{aligned}
$$

$E_{i}, v_{i}$ and $h_{i}$ are the elastic modulus, the Poisson ratio and the thickness of the layer $i$, and the indexes $f$ and $s$ refer to the film and substrate respectively. One has to note that models similar to Stoney model for bending plates measurement give identical results.

\subsection{Fabrication scheme}

This process aims at the fabrication of PDMS based microtubes. Hence, all of the following processes are based on a thin layer of cross-linked PDMS on a layer of poly-4-vynil pyridine (P4VP) - which is used as a low adhesion material on a glass substrate.

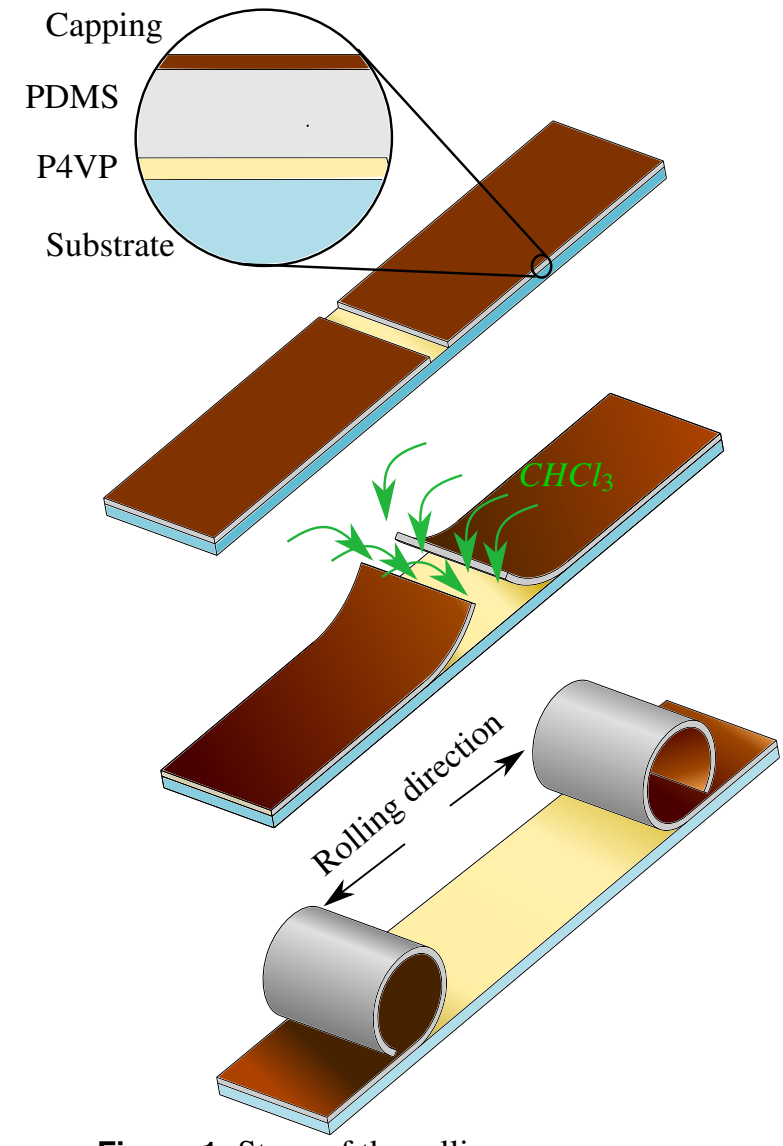

Figure 1. Stage of the rolling process.

Then, the fabrication process must include two important steps. First, one has to introduce some sort of inhomogeneity in the system. Second, a stress that follows this inhomogeneity must be produced.

The former task is done by hardening a PDMS thin film surface by either oxidizing it by oxygen plasma exposure or coating it with a hard material which has a good adhesion to PDMS. Plasma treatment results in a silica-like layer of approximately $100 \mathrm{~nm}^{42}$. In the second case, we chose chitosan - a biocompatible polysaccharide soluble in weak acid-, producing a $270 \mathrm{~nm}$ layer by spin coating.

Subsequently, a cut is done in the film to define the limits of the film to be rolled. The whole system is then exposed to a solvent that swells PDMS but not the hard layer. In our case, chloroform or pentane is used. As illustrated in figure 1, the solvent will preferentially enter through the cut where the free PDMS is exposed and the rolling process occurs.

Once the system is taken out of the solvent, the swelling stimulus quickly disappears. Thus, at large scale (i.e. tubes of several hundreeds micrometers diameter), the system will quickly unroll when taken out of the solvent. However, at smaller scale (i.e. tubes of several tens micrometers diameter), the adhesion of the rolled film on itself is able to hold the tube enclosed.

Many parameters can have a direct influence on the diam- 
eter of the tube in that process. The $n, m$, and $\delta$ parameters in formula 1 can be modified by changing the capping and nature of the solvent. All those aspects are discussed in the next section.

\section{Results and discussion}

\subsection{Influence of the process parameters on the di- ameter}

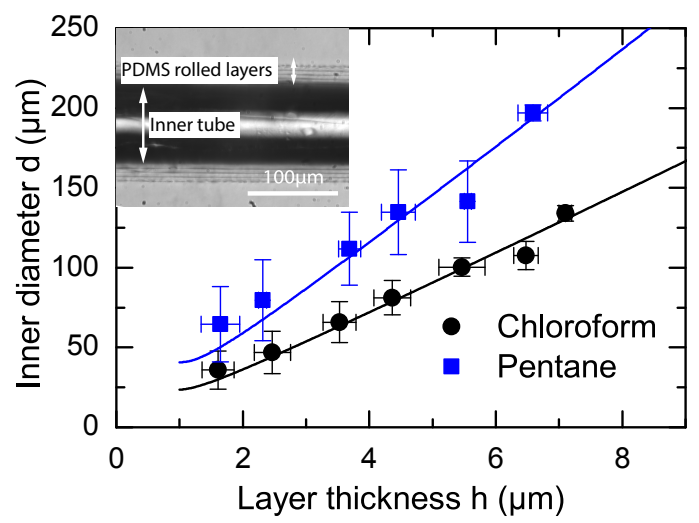

(a) Diameters of oxidized systems obtained with gaseous chloroform and pentane.

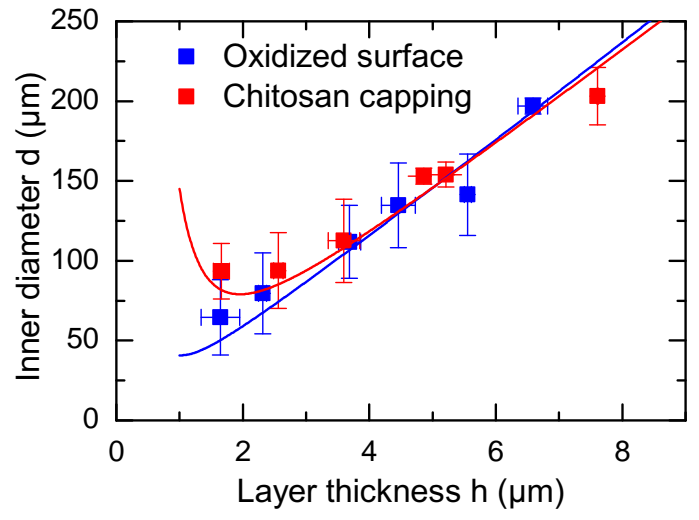

(b) Diameters of oxidized and chitosan coated films rolled in pentane gas.

Figure 2. Inner diameters of rolled tubes as a function of total bilayer thickness for different processes.

We applied this fabrication process to PDMS films with a thickness between 1 and $10 \mu \mathrm{m}$ obtained by spincoating. In order to image the inside of the resulting tube, they are then embedded in a PDMS matrix. Hence, as the optical index does not vary at the external surface of the tube, the inner air/PDMS interfaces can be imaged from above (see inset in figure 2a). This technique is used to measure simultaneously the total thickness of the film and the inner diameter. In some cases, for the thicker films, we noted a variation of the thickness of the different layers, which might be due to a secondary relaxation mode, similar to a wrinkling phenomena. If that was the case, only the thickness of the first layer was measured. If not, the average over all layers was measured.

The inner diameters of tubes is plotted as a function of total thickness of the bilayer in figure 2 for the different processes. In figure $2 \mathrm{a}$, we compare the diameters obtained in gaseous pentane and chloroform while the influence of the capping is compared in figure $2 \mathrm{~b}$. The data was averaged over thickness intervals of $1 \mu \mathrm{m}$. Each point displayed represents the average of 3 to 15 measurements. Hence, the displayed error bars shall be understood as variations of the measured value and not as measurement errors.

For the sake of the confrontation of those results with the predictions of formula 1 , we need to define several parameters:

- The differential strain $\delta$ is just the in-plane swelling ratio of PDMS when the rolling process occurs.

- The thickness of the top layer, its elastic modulus $E$ and Poisson ratio $v$ in unswollen state were already determined in a previous article ${ }^{42}$. For the PDMS substrate we took $E_{P D M S}=2.6 M P a$ and $v=0.5$. For the oxidized PDMS capping layer we used $E=1.5 \mathrm{GPa}$, $v=0.5$ and $h_{f}=175 \mathrm{~nm}$. For the chitosan capping layer we used $E=3 G P a, v=0.3$ and $h_{s}=270 \mathrm{~nm}$.

- As the film swells, the elastic modulus is known to scale as $(1+\delta)^{-143}$.

- Contrary to the thickness of the PDMS film that we measure without solvent, the one in Timoshenko formula has to be the swollen thickness. As the film is initially constrained in both plane direction and PDMS is incompressible, the value to be used is $h_{\text {swollen }}=(1+3 \delta) h_{d r y}$.

The only unknown parameter is the swelling ratio itself. We measured it by recording the swelling ratio of a free PDMS film as a function of exposure time to solvent vapor and comparing it with the time needed of an oxidized pdms film to start rolling. With this method we found that $\delta$ is of the order of 5 to $10 \%$. On figure 2, we tested the hypothesis that $\delta$ is not a function of the thickness $h$ and adjusted its value to fit at best the experimental curve. With values of $\delta$ within our estimation, the formula matches very nicely our experimental result. The parameters used with formula 1 to plot the plain line in figure 2 are summed up in Table 1.

\begin{tabular}{|l|l|l|}
\hline Capping & Oxide & Chitosan \\
\hline $\mathrm{n}$ & $448(1+\delta)$ & $824(1+\delta)$ \\
\hline$h_{f}$ & $175 \pm 45 \mathrm{~nm}$ & $270 \pm 20 \mathrm{~nm}$ \\
\hline \hline Solvent & Chloroform & Pentane \\
\hline$\delta$ & $9.4 \%$ & $5.3 \%$ \\
\hline
\end{tabular}

Table 1. Summary of parameters used for the evaluation of formula 1 for the different processes.

One could note that the value of $\delta$ is below values found in literature ${ }^{44 ; 45}$.Two hypothesis can be made in order to explain this fact. The first one is that the film is only partially 
swollen when the rolling occurs. This hypothesis leads to a strong dependency of $\delta$ in the thickness $h$ that we do not observe, as our results fit better with a constant $\delta$. Hence we reject that hypothesis. The second hypothesis is that the film is saturated in solvent but that for another reason, the saturation limit is lowered. It could be - at least partially explained by the large stress that the rolled system undergoes as it is prevented to swell in-plane ${ }^{46}$ due to the presence of the hard layer. In the stress free case, the swelling ratio is determined by a balance between a mixing free energy of the polymer in the solvent and an elastic energy due to the extension of the polymer chains in the cross-linked network. In the case where in-plane deformations are prevented, the system undergoes forces which make expanding the system even less favourable, preventing the solvent to enter the material. Moreover the PDMS is slightly porous and it has to be added that poromechanics states that solvent will migrates away from compressed areas. As the swelling ratio of the material is directly related to the proportion of solvent it contains, its effective value is lowered. Its prediction in this particular case is a difficult problem, as both the stress field and local solvent concentration are unknown and delicate to measure. This problem remains an open question and is out of the scope of this article.

One last thing to note is for a given $n$, the maximum of curvature in formula 1 is $\kappa=\frac{3}{2} \frac{\delta}{h}$ with $m=1 / \sqrt{n}$. The value of $m$ which results in this largest curvature is in between 0.03 and 0.05 . As the optimum is very shallow, an error of up to a factor 3 on $m$ leads to a change in curvature of only $10 \%$ in this range of $n$. In this work, $m$ spans an experimental range between 0.15 and 0.01 so that those systems are close to the minimal radius of curvature to thickness ratio.

\subsection{Demonstration of the fabrication of a capillary with patterned inner surfaces}

The previously discussed analysis results in the controlled making of tubes with well-defined inner diameters over a wide range, relevant for microfluidic applications. A further step is to pattern the inner surface of the film prior to rolling in order to produce fully patterned cylindrical channels.

\subsubsection{Untreated surface state of rolled-up systems}

For practical applications, the state of the inner surface of the capillary is of utmost importance. We performed AFM measurements of the surface of our systems. The surface of chitosan is uniform with a root mean square roughness $R_{q}=1.85 \mathrm{~nm}$ (data not shown). The plasma oxidized surface has a similar roughness $R_{q}=1.53 \mathrm{~nm}$. However, cracking of the surface occurs as shown on figure 3 . The depth of those cracks is approximately the thickness of the oxide layer and their width is of some microns. In figure 3, we also provide the typical profile of one of those cracks.

For practical reasons, this cracking of the surface can be a problem. However, it can be circumvented by coating it with a supplementary PDMS layer before rolling. As the bending rigidity of a film exhibits a cubic scaling in thickness and

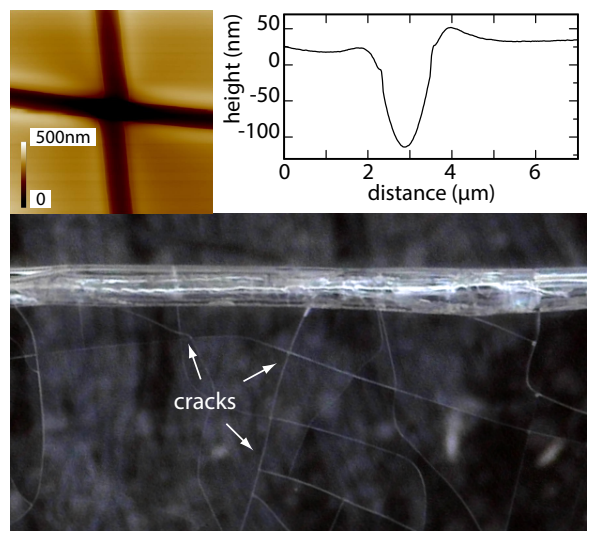

Figure 3. Top AFM image and height profile of a crack on an oxidized PDMS surface. Bottom Optical image of cracks on an oxidized pdms surface during rolling.

linear in elastic modulus, one can easily make the mechanical impact of this film negligible by making it thin and soft for example by diminishing the cross-linker concentration in PDMS-. This step is necessary in particular when very small pattern are to be designed on the surface of the capillary.

\subsubsection{Topographical patterning Topographical patterns}

Chemical patterns

Low adhesion

a) Patterned stamp, treatment produced by soft lithography

b) Pressing the stamp on the film : capped with additional PDMS layer

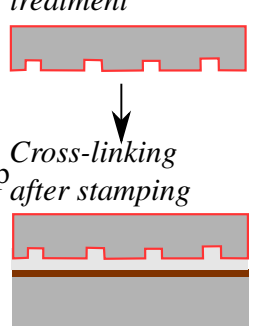

Fluorescent ink

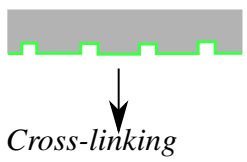

before stamping

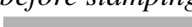
Oxidized PDMS

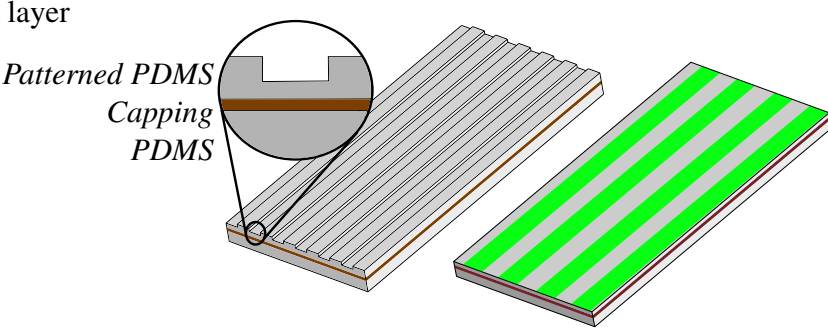

Figure 4. Process steps for the topographic patterning of a system before rolling.

Prior to rolling, the future inner surface of the tube can be functionnalized to obtain 3D patterned systems.

First, we demonstrate an embossing-like method to obtain topographical patterns. In a first step, a PDMS negative replica of the desired patterns is produced by soft lithography that we call the stamp. It is then functionnalized with $(1 \mathrm{H}, 1 \mathrm{H}, 2 \mathrm{H}, 2 \mathrm{H}$-perfluorooctyl)trichlorosilane, which serves as a low adhesion coating. The stamp can then be pressed 
against an uncrosslinked PDMS film so that reticulation occurs against the stamp. The desired structure is obtained once the stamp is removed.

The process is sketched in figure 4 and the obtained capillaries are displayed in figure 5 .

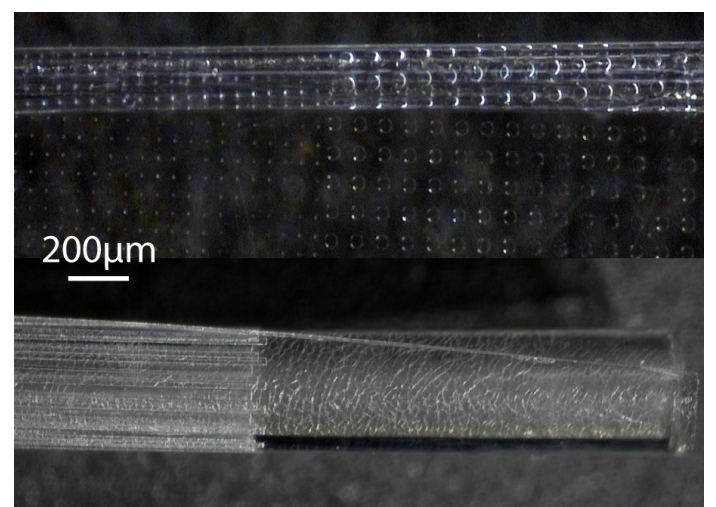

Figure 5. Topographical patterned. Top $2.1 \mu \mathrm{m}$ deep, $10 \mu \mathrm{m}$ and $40 \mu \mathrm{m}$ wide pillars on a $11.5 \mu \mathrm{m}$ rolled film.Bottom $11.5 \mu \mathrm{m}$ deep, $50 \mu \mathrm{m}$ wide grooves on a $48 \mu \mathrm{m}$ rolled film.

\subsubsection{Chemical patterning}

The very similar scheme of microcontact printing can be used to produce chemical patterns (see 4). A PDMS stamp is also produced by soft lithography and inked with a solution of polylysine grafted polyethylene glycol(PLL-g-PEG) labeled with a green fluorescent probe. An oxidized PDMS layer with an additional soft PDMS coating is produced as before. It is exposed briefly to plasma in order to activate the surface without altering its mechanical properties. The stamp is dropped on this surface and left for 5 minutes. Circular holes with diameters of $40 \mu \mathrm{m}$ and $10 \mu \mathrm{m}$ wide lines are stamped on the flat film. The film can then be rolled normally. The resulting systems were imaged with fluorescent microscopy techniques as displayed on figure 6. Rolled-up systems are imaged far from the substrate plane so that only the patterns in the tube are seen.

The pattern in the latter case is only adsorbed to the surface and has a limited resistance in time. However, this method can in principle be adapted to any other microcontact printing method for glassy substrate in order to obtain a covalent bonding of the pattern as in Abdullah et al. ${ }^{47}$.

\section{Conclusion}

Spontaneous curvature is induced in capped PDMS thin film by selective solvent swelling. That method is used as a nonlithographic mean to obtain self-rolled micro-capillaries with an inner diameter between 20 and $200 \mu \mathrm{m}$. The obtained diameter as a function of film thickness was optically measured for different solvent - chloroform and pentane - and capping PDMS oxide and chitosan -.

Those results are confronted to Timoshenko model. Although one parameter is only known in term of order of

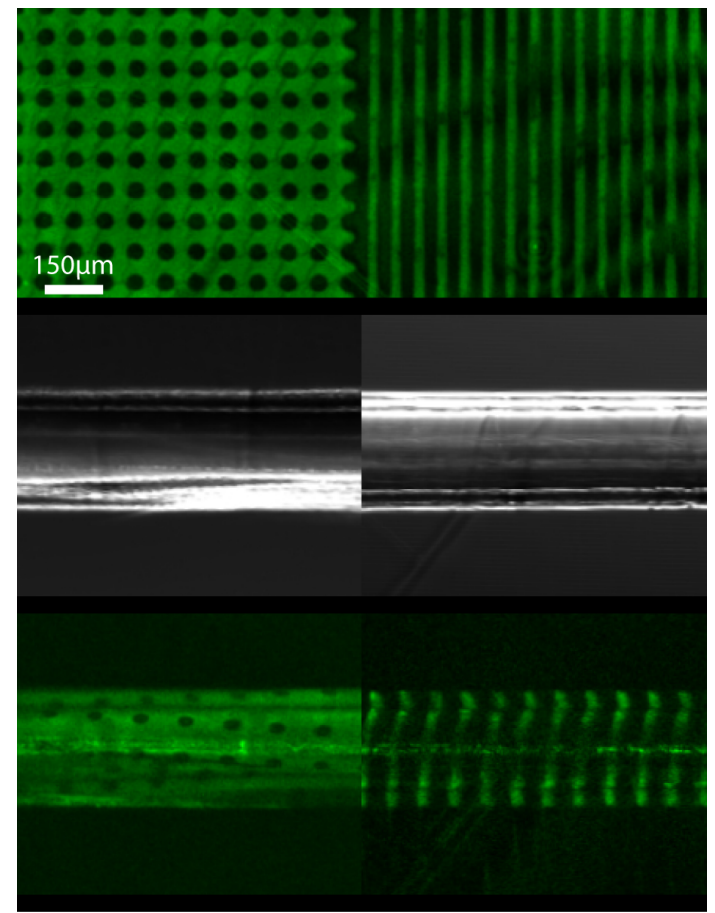

Figure 6. Fluorescence images of stamped system Top before and Bottom after rolling. Middle Rolled up system in bright field imaging.

magnitude, the predictive ability of the model seems very satisfactory.

The striking feature of that system is that the future inner surface of those tubes is initially accessible so that it can be properly characterized or functionnalized before rolling. The different surfaces state resulting from the different capping were imaged by AFM. Examples of topographical and chemical patterning were performed as a concept demonstration.

In the context of microfluidic, soft lithographic methods are widely used because designs based on channel shapes - $\mathrm{T}$ or Y junctions, flow focusing - are easy to fabricate. However, techniques requiring further surface specialization - grooved surface, local change in wetability, cell adsorption - can require a lot more efforts. On the contrary, self-rolled polymer capillaries present great hope for the trivial design of specialized capillaries, but only tubular channels are obtained. Hence, the establishment of hybrid methods taking advantage of both techniques could be a new route to broaden the possibilities of microfluidic chip design. Such methods are currently under investigation.

\section{Experimental}

\subsection{Material}

PDMS elastomer was purchased from Dow Corning (Sylgard 184). P4VP, chitosan, silane and solvents were purchased from Sigma Aldrich, polylysine $(20 \mathrm{kDa})$ grafted with polyethylene glycol $(2 \mathrm{kDa})$ and labeled with fluorescein isothiocyanate (PLL-g-PEG/FTIC) was purchased from Susos. 


\subsection{Film fabrication}

Glass substrates are cleaned by UV-Ozone, plasma and acid technique ${ }^{48}$ to get reproducible wetting and surface state. A solution of P4VP in ethanol $\left(1\right.$ g. $\left.L^{-1}\right)$ is spin coated on the substrate at $3000 R P M$ for 30 seconds with an acceleration of 500 RPM.s ${ }^{-1}$.

Sylgard PDMS is mixed with the furnished cross-linker with a ratio 10:1. After degazing, this mixture was either directly spin coated for the fabrication of layers thicker than $10 \mu \mathrm{m}$ or further diluted in toluene in proportion $3: 1,1: 1$ and $3: 1$ in order to reduce the viscosity of the solution for the fabrication of thinner layers.

Thicker films are obtained by spin-coating on the substrate with three steps. First, a step at 500 RPM for 30 seconds is done to spread PDMS uniformly on the substrate. Second, a step at various speeds for 5 minutes takes place in order to adjust the film thickness (typically, with a speed of 3000 RPM, a thickness of $12.8 \mu \mathrm{m}$ is obtained. Finally for thicker films, a 2 second pulse at $5000 R P M$ is used to remove the edge beads. The acceleration is 3000 RPM.s s $^{-2}$

Thinner films are obtained by spin-coating of diluted PDMS on the substrate at various speeds between $3000 R P M$ and $10000 R P M$ for one minute with an acceleration on 500 RPM.s ${ }^{-1}$.

The samples are then placed on a hot plate at $150{ }^{\circ} \mathrm{C}$ for 15 minutes to ensure a quick evaporation of toluene and a complete cross-linking of the material.

\subsection{Capping fabrication}

Oxidized PDMS samples are prepared in a Harrick's plasma cleaner. Oxygen is supplied to the chamber where the pressure is regulated by the equilibrium between the oxygen entrance flow rate (monitored with a microvalve) and a fixed outflow. The pressure is set to $4.10^{-1}$ mbar and the plasma is powered on at $29.6 \mathrm{~W}$ for 40 minutes. An additional PDMS layer can eventually be spin-coated above this oxide layer as in 4 . The PDMS to cross-linker ratio used is 1:20 and its typical thickness is one third to one fourth of the carrier film thickness.

Chitosan is dissolved in chlorhydric acid $(p H=1)$. The dissolution process is slow and can be accelerated by extended sonication. In order to prepare chitosan on PDMS samples, those are exposed to plasma for one minute to make the surface hydrophilic. The chitosan solution can then be spin-coated on the substrate at $500 R P M$ for one minute with an acceleration on $500 R P M . s^{-1}$. The samples are then dried for one hour at $80^{\circ} \mathrm{C}$ on a hot plate.

\subsection{Rolling}

The film is first cut in order to release the borders of the future tube. It is then placed in a closed glass petri dish filled with a few milliliters of solvent. Two small pillars maintain the system a few millimeters away from the solvent, the film directly facing its surface. In those conditions, the rolling occurs within minutes. For thicker systems, the system often rolls back once taken of the solvent. The stability of the tube can be increased by a liquid phase annealing in the same solvent for a few hours which makes the deformation only partially reversible. This annealing step was never performed for the measurement of inner diameter as a function of experimental parameters.

\subsection{Topographic patterning of a PDMS film}

A PDMS $5 \mathrm{~mm}$ stamp is fabricated by conventional soft lithography techniques ${ }^{49}$. The surface of the stamp is activated with oxygen plasma for 2 minutes. The stamp is then placed for 12 hours in a closed 4 inches wide petri dish with $10 \mu \mathrm{L}$ of $(1 \mathrm{H}, 1 \mathrm{H}, 2 \mathrm{H}, 2 \mathrm{H}$-perfluorooctyl)trichlorosilane. Before its use, The stamp is degazed at 0.1 mbar for at least 10 minutes

A PDMS film of desired thickness is obtained as above Before the cross-linking of the PDMS occurs, the stamp is carefully applied against the film so that large bubbles are avoided (small bubbles will disappear if the stamp was properly degazed). The system and stamp are then placed on a hot plate at $80^{\circ} \mathrm{C}$ for an hour. In order to prevent unsticking of the stamp due to thermal effects, a weight of 500 grams is put on the sample during the reticulation process. After cooling down of the system the stamp is carefully removed. This step is tricky has the fragile film is lowly adhesive on both the substrate and the stamp. This step is greatly facilitated by adding a few drops of ethanol at the edge of the stamp which instantly causes the delamination of the stamp. However, one should be careful that ethanol does not reach the substrate as P4VP is soluble in ethanol.

Two cases are described in this paper. The first case is to stamp the first layer of PDMS which can then be oxidized and rolled as above. The second case is to stamp an additional thinner and softer PDMS layer on an oxidized ready to roll layer. It can then directly be cut and rolled as above. The former method is more suitable for large systems, while the latter is essential to design very small structures which would be damaged by exposure to plasma.

\subsection{Microcontact printing}

A stamp was designed with soft lithography techniques ${ }^{49}$. A few drops of aqueous solution of PLL-g-PEG/FTIC $0,1 \mathrm{mg} / \mathrm{L}$ are poured on the stamped and spread by pressing a glass slide on top of it. After 5 minutes of infusion, the stamp is dried with nitrogen until no solvent remains. A ready to roll film with an additional layer of soft PDMS is designed as above. It exposed to oxygen plasma with an for 2 minutes. The stamp is dropped on the activated surface and left for 10 minutes. The system can then be rolled as above.

\subsection{Size measurements and imaging}

Tube were encased in PDMS and optically imaged with an optical transmission microscope. The images were treated with imageJ software to measure simultaneously the thickness of the film and the diameter of the tube. Patterned systems in inset of figure 2a were imaged with an Dino-lite numerical microscope. Both the thickness of the film and the 3dimensionnal shape of the patterns were measured with an optical interferometer. The surface state of the systems were 
imaged with an AFM Dimension V (Digital Instruments / Veeco-Bruker, Santa Barbara, CA, USA) in contact mode. Fluorescence was imaged with an Olympus Fluoview FV1000 inverted confocal microscope.

\section{Acknowledgments}

This work was supported by the French National Research Agency, Grant no ANR-13-IS09-0002.

\section{References}

[1] E. J. Smith, W. Xi, D. Makarov, I. Monch, S. Harazim, V. A. Bolanos Quinones, C. K. Schmidt, Y. Mei, S. Sanchez and O. G. Schmidt, Lab Chip, 2012, 12, 19171931.

[2] J. Zang and F. Liu, Nanotechnology, 2007, 18, 405501.

[3] J. Fritz, analyst, 2008, 133, 855-863.

[4] R. Raiteri, M. Grattarola, H.-J. Butt and P. Skládal, Sensors and Actuators B: Chemical, 2001, 79, 115-126.

[5] S. Schwaiger, A. Rottler and S. Mendach, Advances in OptoElectronics, 2012, 2012,.

[6] W. Huang, X. Yu, P. Froeter, R. Xu, P. Ferreira and X. Li, Nano letters, 2012, 12, 6283-6288.

[7] X. Li, Advances in Optics and Photonics, 2011, 3, 366387.

[8] D. J. Thurmer, C. Deneke, Y. Mei and O. G. Schmidt, Applied physics letters, 2006, 89, 223507.

[9] A. Prinz, V. Y. Prinz and V. Seleznev, Microelectronic engineering, 2003, 67, 782-788.

[10] P. Froeter, Y. Huang, O. V. Cangellaris, W. Huang, E. W. Dent, M. U. Gillette, J. C. Williams and X. Li, ACS nano, 2014, 8, 11108-11117.

[11] M. Yu, Y. Huang, J. Ballweg, H. Shin, M. Huang, D. E. Savage, M. G. Lagally, E. W. Dent, R. H. Blick and J. C. Williams, ACS nano, 2011, 5, 2447-2457.

[12] J. Deng, H. Ji, C. Yan, J. Zhang, W. Si, S. Baunack, S. Oswald, Y. Mei and O. G. Schmidt, Angewandte Chemie, 2013, 125, 2382-2386.

[13] V. Luchnikov, O. Sydorenko and M. Stamm, Advanced Materials, 2005, 17, 1177-1182.

[14] K. Kumar, V. Luchnikov, B. Nandan, V. Senkovskyy and M. Stamm, European Polymer Journal, 2008, 44, 41154121.

[15] A. Egunov, J. Korvink and V. Luchnikov, Soft matter, 2016, 12, 45-52.
[16] V. Luchnikov, M. Stamm et al., Macromolecular rapid communications, 2011, 32, 1943-1952.

[17] S. Zakharchenko, E. Sperling and L. Ionov, Biomacromolecules, 2011, 12, 2211-2215.

[18] L. Ionov, Journal of Materials Chemistry, 2012, 22, 19366-19375.

[19] Y. Mei, A. A. Solovev, S. Sanchez and O. G. Schmidt, Chemical Society Reviews, 2011, 40, 2109-2119.

[20] A. E. Shyer, T. Tallinen, N. L. Nerurkar, Z. Wei, E. S. Gil, D. L. Kaplan, C. J. Tabin and L. Mahadevan, Science, 2013, 342, 212-218.

[21] J. Genzer and J. Groenewold, Soft Matter, 2006, 2, 310323.

[22] S. Cai, D. Chen, Z. Suo and R. C. Hayward, Soft Matter, 2012, 8, 1301-1304.

[23] F. Weiss, S. Cai, Y. Hu, M. K. Kang, R. Huang and Z. Suo, Journal of Applied Physics, 2013, 114, 073507.

[24] V. Y. Prinz, V. Seleznev, A. Gutakovsky, A. Chehovskiy, V. Preobrazhenskii, M. Putyato and T. Gavrilova, Physica E: Low-dimensional Systems and Nanostructures, 2000, 6, 828-831.

[25] R. Songmuang, C. Deneke and O. Schmidt, Applied physics letters, 2006, 89, 223109.

[26] S. Yang, K. Khare and P.-C. Lin, Advanced Functional Materials, 2010, 20, 2550-2564.

[27] V. Luchnikov and M. Stamm, Physica E: Lowdimensional Systems and Nanostructures, 2007, 37, 236240.

[28] K. Kumar, B. Nandan, V. Luchnikov, E. B. Gowd and M. Stamm, Langmuir, 2009, 25, 7667-7674.

[29] L. P. Chia Gómez, P. Bollgruen, A. I. Egunov, D. Mager, F. Malloggi, J. G. Korvink and V. A. Luchnikov, Lab on a Chip, 2013, 13, 3827.

[30] A. D. Stroock, S. K. Dertinger, G. M. Whitesides and A. Ajdari, Analytical Chemistry, 2002, 74, 5306-5312.

[31] E. Martinez, E. Engel, J. Planell and J. Samitier, Annals of Anatomy-Anatomischer Anzeiger, 2009, 191, 126-135.

[32] A. Béduer, L. Vaysse, E. Flahaut, F. Seichepine, I. Loubinoux and C. Vieu, Microelectronic Engineering, 2011, 88, 1668-1671.

[33] R. Marie, J. P. Beech, J. Vörös, J. O. Tegenfeldt and F. Höök, Langmuir, 2006, 22, 10103-10108.

[34] S. Béfahy, P. Lipnik, T. Pardoen, C. Nascimento, B. Patris, P. Bertrand and S. Yunus, Langmuir, 2010, 26, 33723375 . 
[35] T. Nishino, R. Matsui and K. Nakamae, Journal of Polymer Science Part B: Polymer Physics, 1999, 37, 11911196.

[36] H. Li, PhD thesis, UNIVERSITY OF MINNESOTA, 2012.

[37] G. G. Stoney, Proceedings of the Royal Society of London. Series A, Containing Papers of a Mathematical and Physical Character, 1909, 82, 172-175.

[38] S. Timoshenko, JOSA, 1925, 11, 233-255.

[39] N. Salamon and C. B. Masters, International journal of solids and structures, 1995, 32, 473-481.

[40] S. Alben, B. Balakrisnan and E. Smela, Nano letters, 2011, 11, 2280-2285.

[41] N. Guyot, Y. Harmand and A. Mézin, International journal of solids and structures, 2004, 41, 5143-5154.

[42] B. Sarrazin, R. Brossard, P. Guenoun and F. Malloggi, Soft Matter, 2016, 12, 2200-2207.

[43] M. Rubinstein and R. Colby, Polymers physics, Oxford Oxford, UK, 2003, vol. 767.

[44] J. N. Lee, C. Park and G. M. Whitesides, Analytical chemistry, 2003, 75, 6544-6554.

[45] C. Rumens, M. A. Ziai, K. Belsey, J. C. Batchelor and S. J. Holder, Journal of Materials Chemistry C, 2015, 3, 10091-10098.

[46] K. Dusek, A. Choukourov, M. Duskova-Smrckova and H. Biederman, Macromolecules, 2014, 47, 4417-4427.

[47] N. H. Abdullah, W. A. W. A. Bakar, R. Hussain, M. B. Bakar, M. Mohamed, M. K. A. A. Razab and J. H. van Esch, ARPN, 2006, 10, 9538-9543.

[48] W. Kern, Journal of the Electrochemical Society, 1990, 137, 1887-1892.

[49] Y. Xia and G. M. Whitesides, Angewandte Chemie International Edition, 1998, 37, 550-575. 